\title{
Familial juvenile hyperuricaemic nephropathy (FJHN): linkage analysis in 15 families, physical and transcriptional characterisation of the FJHN critical region on chromosome $16 \mathrm{p} 11.2$ and the analysis of seven candidate genes
}

\author{
Blanka Stibůrková ${ }^{1}$, Jacek Majewski ${ }^{2}$, Kateřina Hodaňová ${ }^{1}$, Lenka Ondrová ${ }^{1}$, \\ Markéta Jeřábková ${ }^{1}$, Marie Zikánová ${ }^{1}$, Petr Vylet'al ${ }^{1}$, Ivan Šebesta ${ }^{3}$, Anthony Marinaki ${ }^{4}$, \\ Anne Simmonds ${ }^{4}$, Gert Matthijs ${ }^{5}$, Jean-Pierre Fryns ${ }^{5}$, Rosa Torres ${ }^{6}$, Juan García Puig ${ }^{7}$, \\ Jurg Ott ${ }^{2}$ and Stanislav Kmoch*,1
}

\begin{abstract}
${ }^{1}$ Center for Integrated Genomics, Institute for Inherited Metabolic Disorders, Charles University 1st School of Medicine and General Faculty Hospital Prague, Czech Republic; ${ }^{2}$ Laboratory of Statistical Genetics, Rockefeller University, New York, NY, USA; ${ }^{3}$ Department of Clinical Biochemistry, Charles University 1st School of Medicine and General Faculty Hospital Prague, Czech Republic; ${ }^{4}$ Purine Research Unit, GKT, Guy's Hospital, London, UK; ${ }^{5}$ Center for Human Genetics, University of Leuven, Leuven, Belgium; ${ }^{6}$ Department of Clinical Biochemistry, La Paz Hospital, Madrid, Spain; ${ }^{7}$ Division of Internal Medicine, La Paz Hospital, Madrid, Spain
\end{abstract}

Familial juvenile hyperuricaemic nephropathy (FJHN) is an autosomal dominant renal disease characterised by juvenile onset of hyperuricaemia, gouty arthritis, and progressive renal failure at an early age. Recent studies in four kindreds showed linkage of a gene for FJHN to the same genomic interval on chromosome 16p11.2, where the gene for the phenotypically similar medullary cystic disease type 2 (MCKD2) has been localised. In this study we performed linkage analysis in additional 15 FJHN families. Linkage of FJHN to 16p11.2 was confirmed in six families, which suggests that, in a large proportion of FJHN kindreds, the disease is likely to be caused by a gene or genes located outside of 16p11.2. Haplotype analysis of the new and previously analysed families provided two non-overlapping critical regions on 16p11.2-FJHN1, delimited by markers D16S499-D16S3036 and FJHN2, delimited by markers D16S412-D16S3116. Considering MCKD2 to be a distinct molecular entity, the analysis suggests that as many as three kidney disease genes may be located in close proximity on 16p11.2. From genomic databases we compiled integrated physical and transcription maps of whole critical genomic region in which 45 known genes and 129 predicted loci have been localised. We selected, analysed and found no pathogenic mutations in seven candidate genes. The linkage and haplotype analysis reported here demonstrates the genetic heterogeneity of FJHN. The report of integrated physical and mostly in-silico predicted transcription maps of the FJHN critical region provides a basis for precise experimental annotation of the current transcript map, which is essential for final identification of the FJHN gene(s). European Journal of Human Genetics (2002) 11, 145 -154. doi:10.1038/sj.ejhg.5200937

\footnotetext{
*Correspondence: Stan Kmoch, Center for Integrated Genomics, Institute for Inherited Metabolic Disorders, Ke Karlovu 2, 12800 Prague 2, Czech Republic. Tel: 4202 24967691; Fax: 4202 24919392;

E-mail: SKMOCH@LF1.CUNI.CZ

Received 13 August 2002; revised 12 November 2002; accepted 15 November 2002
}

Keywords: FJHN; MCKD; hyperuricaemia; gout; nephropathy; chromosome 16p11.2 


\section{Introduction}

Familial juvenile hyperuricaemic nephropathy (FJHN), (OMIM 162000), is an autosomal dominant renal disease characterised by juvenile onset of hyperuricaemia, gouty arthritis, and progressive renal failure. The disease was first noted by Duncan and Dixon in $1960 .{ }^{1}$ More than 50 kindred originating from various ethnic groups - Caucasians (for review see ${ }^{2}$ ) and, ${ }^{3-11}$ Hungarian, ${ }^{12}$ Japanese, ${ }^{13,14}$ Chinese, ${ }^{15}$ Polynesian $^{16}$ and recently also Finnish ${ }^{17}$ and Chilean ${ }^{18}$ have been described so far.

The pathogenesis and molecular basis of FJHN is unknown. Evidence that hyperuricaemia associated with the urate hypoexcretion is the primary event of the nephropathy was provided by McBride et al, ${ }^{19,20}$ who found normal renal function in $42 \%$ of children biochemically proven to have FJHN. In accordance with these results, the disease seems to result from a defect of uric acid tubular transport. Candidate proteins could be either voltage-sensitive urate transporters such as hUAT1 and hUAT2, ${ }^{21}$ suggested urate/anion exchangers ${ }^{22}$ or other proteins which may be involved in forming, regulating, processing, trafficking, and cell surface stability of structurally functional urate channel multimeric complex. In addition to the tubular transport defect hypothesis, Puig et $a l^{7}$ suggested that uric acid underexcretion and hyperuricaemia may be a consequence of a primary renal hemodynamic disruption.

Furthermore, the clinical, biochemical and histological examinations observed in FJHN are similar to those observed in diseases belonging to nephronophthisis-medullary cystic kidney disease complex, ${ }^{23}$ and it was postulated that connection between FJHN and these diseases, particularly medullary cystic kidney disease type 2 (MCKD2), may exist. ${ }^{11,24,25}$

Recent studies, ${ }^{26-28}$ one of them long-term, ${ }^{28}$ have demonstrated the beneficial effect of allopurinol in ameliorating the progression of the renal disease. However the beneficial effect of allopurinol is controversial. ${ }^{29}$ Others suggest that in patients with early stage nephropathy blockade of the renin-angiotensin system may delay the progression of renal insufficiency. ${ }^{30,31}$ These findings underscore the importance of pre-symptomatic diagnosis, especially in children. Pre-symptomatic detection enabling diagnosis and the commencement of early treatment is vital for affected families and will also result in preventing or delaying dialysis and ultimately, renal transplantation.

Identifying the genetic defect is essential to facilitate such a diagnosis and replace the non-specific biochemical tests that depend on the accuracy of sample collection. Identifying the hypothetical defective urate transporter in FJHN may have broader relevance to a much more common disease of defective urate transport - the underexcreting gout ('primary' gout) affecting middle-aged males. It is possible that mutation and/or polymorphism in genes causing FJHN may also be implicated there.
Recently we $\mathrm{e}^{32}$ and others ${ }^{11,33}$ localised a gene for FJHN in four kindreds to chromosome 16p11.2, and found evidence of genetic heterogeneity of the disease. Interestingly, the FJHN locus was mapped to the same genomic interval where the gene for medullary cystic disease type 2 (MCKD2) (OMIM 603860), has been localised. ${ }^{25,34}$ Clinical and biochemical similarities and genomic co-localisation of both loci suggest that FJHN and MCKD2 may be a single disease entity. Alignment of all critical regions detected in these studies, however, provides two non-overlapping genomic regions delimited by markers D16S499-D16S3036 and D16S403-D16S3116, ${ }^{11}$ and keeps the issue of the distinctness of FJHN and MCKD loci open.

In this study we performed linkage analysis for FJHN in additional 15 families, and confirmed in six the previous observations of linkage of FJHN to $16 \mathrm{p} 11.2$, which suggests, that in a large proportion of FJHN kindreds, the disease is likely to be caused by a gene or genes located outside of 16p11.2. Haplotype analysis showed that two families mapped in this study might be aligned to the D16S499D16S3036 interval only whereas the rest of the families may be aligned with both intervals. Considering MCKD2 as a distinct molecular entity, we hypothesise that as many as three genes located in close proximity on $16 \mathrm{p} 11.2$ may exist. From genomic databases we therefore compiled integrated physical and transcription maps of whole implicated genomic region (D16S499-D16S3116). We selected, analysed and excluded seven candidate genes. The results should facilitate efficient candidate gene selection and in combination with high throughput sequencing and functional genomic studies should help to resolve the molecular basis of FJHN/MCKD disease(s) in the future.

\section{Methods}

\section{Patients}

In total, 15 FJHN families (118 individuals of which 58 were affected) were enrolled in this study. Ten families are from Great Britain (GB1-10), three from Spain (E1-3) and two from Belgium (BE1 and BE2). The diagnosis of FJHN was based on the following criteria: autosomal dominant inheritance, hyperuricaemia, gouty arthritis which progress to renal failure. Clinical and biochemical data of the GB and Spanish patients have been described in detail previously. 2,7,20,24,27,28

In family BE1, the first symptoms in the affected individuals were mild anaemia (Hb levels $10 \mathrm{~g} \%$ ), slowly progressive renal failure and hyperuricaemia from the age of 10 years. The patients had small, echogenic kidneys on renal echography. Terminal renal failure treated with renal transplantation developed at the age of 60 years in one of the affected males (DII.1).

In family BE2, three affected brothers developed the first symptoms of hyperuricaemia and gouty arthritis after the age of 30 years. Allopurinol treatment started at the first 
symptoms. Between 45 and 50 years of age, the first symptoms of renal failure were registered (creatinin: $2.59 \mathrm{mg} /$ $100 \mathrm{ml}$ ). Renal echography showed small hyperechogenic kidneys. Between 55 and 60 years of age, symptoms of arterial hypertension and progressive preterminal renal failure (creatinin levels of $5.36 \mathrm{mg} / \mathrm{ml}$ in the proband) appeared.

\section{Genotype analysis}

Genotyping was performed as previously described, ${ }^{32}$ using a set of 13 microsatellite markers spanning the $13 \mathrm{cM}$ of the critical region on chromosome 16p11.2. The marker order was as follows: cen- D16S499-D16S501-D16S3056D16S3041-D16S3036-D16S3046-D16S403-D16S412-D16S3130D16S417-D16S420-D16S3113-D16S401-tel and was based on the Marshfield Genetic Map.

\section{Linkage analysis}

Genotyping data were screened for errors using the PEDCHECK program. ${ }^{35}$ Two point and multipoint linkage analyses, along with determination of the most likely haplotypes, were performed using the Allegro program. ${ }^{36}$ The analysis was carried out under the assumption of a dominant mode of inheritance with a 95\% penetrance, $5 \%$ phenocopy rate and 0.001 frequency of the disease allele. Marker allele frequencies were estimated from the families used in this study using the program Pedmanager http://www.genome.wi.mit.edu/ftp/pub/software/pedmanag).

\section{Integrated genetic, physical and transcript map}

Genetic and STS markers were positioned on human genomic contigs by searching in genome databases MapViewer, builds 27-29, (http://www.ncbi.nlm.nih.gov/), Ensembl - V1.2.0.(http://www.ensembl.org/ ) and Human Genome Working Draft at UCSC, August 2001 freeze, (http://genome.ucsc.edu/). To fill the existing gaps, contig ends BAC sequences were BLASTed against the Homo sapiens genomic contig sequences, high-throughput genomic sequencing (htgs) and BAC ends sequences sections of the NCBI database. Gene content was obtained either directly from annotated contigs or using the BLAST queries of unfinished genomic sequences against dbEST and protein databases.

\section{Candidate gene analysis}

Individual genes were evaluated according to knowledge or assumption of their function, evaluation of their expression profiles, and analysis of hypothetical functional domains. Upstream promotor region sequence, genomic organisation, and genomic sequence of candidate genes were obtained using pairwise BLASTN analysis of the cDNA with corresponding BAC sequences. Genomic fragments covering promotor region (about $500 \mathrm{bp}$ upstream from the most cDNA $5^{\prime}$ end) and all of the exons and intron-exon bound- aries were PCR amplified from genomic DNA and sequenced as previously described. ${ }^{37}$ All candidate genes were analysed in one proband and one unaffected family member in each family. Genetic variations were screened against SNP and UNIGENE databases.

\section{Results}

Linkage analysis

The results of a multipoint linkage analysis are shown in Table 1 and graphically in Figure 1 . We calculated the HLOD score, allowing for genetic heterogeneity by maximising the score over the parameter, representing the proportion of families in the sample demonstrating genetic linkage of the disease to the region of interest. A maximum HLOD score of 1.6137 was obtained at markers D16S3041D16S3036 for $\alpha=0.374$. In order to determine the statistical significance of this finding, one needs to subtract 0.3 from the maximum HLOD value, as recommended by Terwilliger and $\mathrm{Ott},{ }^{38}$ to adjust for maximisation of the score over two parameters (and the recombination fraction). It is then possible to use the well-known property of asymptotic convergence of the $\chi^{2}$ distribution and two times natural logarithm of the odds ratio. After the adjustment, HLOD of 1.6137 corresponds to a $\chi^{2}$ of 6.05 (one tail $P=0.007$ ). Thus our finding confirms the previous observations of linkage of FJHN to $16 \mathrm{p} 11.2 .^{11,32,33}$

However, linkage to $16 \mathrm{p} 11.2$ may be excluded at the LOD $=-2$ level for families BE1 and GB4, while some of the other families also have negative LOD scores (without reaching the -2 level) throughout the candidate region. The value of $\alpha=0.374$ implies that genetic linkage between FJHN and $16 \mathrm{p} 11.2$ exists in only $37 \%$ of the analysed families. Thus, while confirming linkage of FJHN to the region, our analysis provides further evidence of heterogeneity and shows that a large proportion of FJHN cases are likely to be caused by genes located outside of $16 \mathrm{p} 11.2$.

\section{Haplotype analysis}

Haplotype analysis showed consistent segregation of $16 \mathrm{p} 11.2$ haplotypes and the FJHN phenotype in six of the families (BE2, GB2, GB3, GB8, E1, E3). Thus, six out of 15 families (40\%) appear to have haplotypes consistent with linkage to FJHN, providing another estimate of the degree of genetic heterogeneity of the disease. However, the haplotypes segregating within individual families are not shared across families, nor is any single allele at any of the linked markers shared across families.

Pedigrees of these families are shown in Figure 2. We have aligned detected recombination events with critical regions delimited by haplotype analyses in all the FJHN and MCKD families linked to chromosome $16 \mathrm{p} 11.2$ region so far (Figure 3). This alignment provides two genetic intervals - D16S499-D16S3036 (FJHN1) and D16S412-D16S3116 (FJHN2) delimited by critical recombinations detected in Belgian $^{11}$ and Japanese ${ }^{33}$ families. From eight haplotypes 
Table 1 Maximum LOD scores within the D16499D16S3046 interval for individual families

\begin{tabular}{ll}
\hline Family & $\begin{array}{l}\text { Maximum multipoint } \\
\text { LOD score }\end{array}$ \\
\hline BE1 & $-4.9266^{\mathrm{a}}$ \\
BE2 & $0.8437^{\mathrm{b}}$ \\
GB1 & $-0.3521^{\mathrm{c}}$ \\
GB2 & $0.2919^{\mathrm{b}}$ \\
GB3 & $1.4520^{\mathrm{b}}$ \\
GB4 & $-5.9092^{\mathrm{a}}$ \\
GB5 & $-0.2848^{\mathrm{c}}$ \\
GB6 & $0.1155^{\mathrm{c}}$ \\
GB7 & $-0.4721^{\mathrm{c}}$ \\
GB8 & $0.5862^{\mathrm{b}}$ \\
GB9 & $0.2911^{\mathrm{c}}$ \\
GB10 & $-1.1788^{\mathrm{c}}$ \\
E1 & $0.5872^{\mathrm{b}}$ \\
E2 & $-1.0602^{\mathrm{c}}$ \\
E3 & $0.8346^{\mathrm{b}}$ \\
\hline
\end{tabular}

${ }^{\mathrm{a}}$ Families excluded for linkage to $16 \mathrm{p} 11.2$. ${ }^{\mathrm{b}}$ Families segregated haplotypes fully consistent with linkage. 'Families with haplotypes inconsistent with linkage.

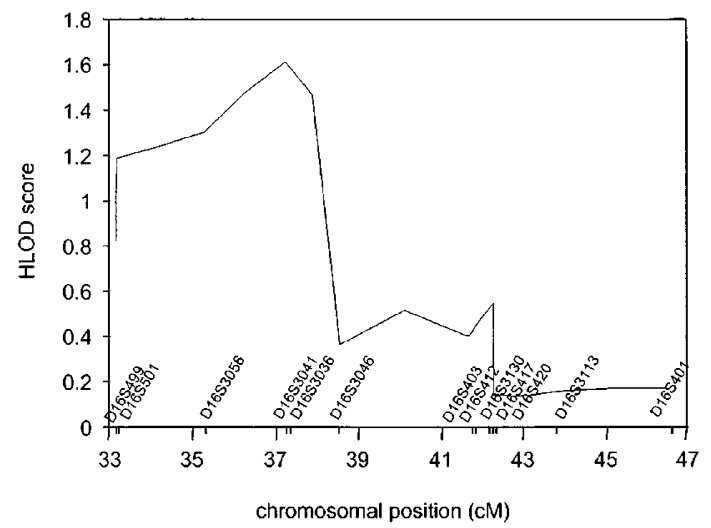

Figure 1 Multi-point overlapping HLOD score analysis of the markers in the linked region on chromosome 16.

defined in this and previous study, ${ }^{32}$ two haplotypes overlap with the FJHN1 region only. The remaining six haplotypes, as well as two haplotypes defined in MCKD2 studies, ${ }^{25,34}$ overlap with both FJHN1 and FJHN2 regions.

\section{Integrated genetic, physical and transcript map}

In silico analysis positioned all of the Marshfield genetic markers used for haplotype analysis to four genomic contigs - NT_010393, NT_024776, NT_010441 and NT_10604. Using the BAC end sequence content analysis, three BAC clones closing the gaps between individual contigs were identified. This effort, summarised in Figure 4 , delimited approximately $12.6 \mathrm{Mb}$ region on chromosome 16p11.2. Based on the NCBI contig assembly and annotation this region contains altogether 45 known genes and 129 predicted loci.

\section{Candidate gene analysis}

The following genes were considered and analysed as potential candidates for FJHN.

MIR16 (RGS16-INTERACTING MEMBRANE PROTEIN), UniGene Cluster Hs.107014 is an integral membrane glycoprotein with putative phosphodiesterase enzymatic activity, localised on the liver and kidney plasma membrane where it may play important roles in lipid metabolism and $\mathrm{G}$ protein signalling. ${ }^{39}$ Sequence analysis showed heterozygous transition c1305A-G located in 3'UTR in one of the patients. This polymorphism was reported in dBSNP previously.

GPRC5B (G protein-coupled receptor) UniGene Cluster Hs. 242407 is a member of type 3 GPRC family. The specific function of this protein is unknown; however, it may mediate the cellular effects of retinoic acid on the $G$ protein signal transduction cascade. ${ }^{40}$ Seven sequence variants were detected in GPRC5B. Three single base pair deletions were present homozygously in both patients and controls suggesting the sequencing error in genome databases. Three heterozygous single base pair substitutions: g1756A-G, g11749G-A and g1086T-C were found in non-coding regions of both patients and controls. Homozygous silent transition c804C-T was found in a control.

Ks-1 (kidney specific protein 1, Sa protein), LOC123876, is a human homologue of rat hypertension associated protein which exhibits a unique tissue-specific expression exclusively in mature kidneys and livers. A recent study identified the protein as a medium-chain acyl-CoA synthetase. ${ }^{41}$ Six sequence variants were detected in the Ks-1 gene. Two transitions, c340G-A and c428T-C, changing 114GlnLys and 143Leu-Ser, respectively, transversion g1166C-A, and a homozygous single base pair deletion g3385delT were found in both patients and controls. Two heterozygous transitions, silent c438G-A (146 Leu-Leu) and g3384C-T, were found in both patients and controls.

Ks-2 (kidney specific protein 2), MACS1 (Butyryl Coenzyme A synthetase 1), UniGene Cluster Hs.98732, is localised in an opposite transcriptional direction in close proximity to the Ks-1 gene. Both rat and mouse homologues showed kidney and liver specific gene expression. ${ }^{41}$ Three sequence variants, silent transitions c45T-C and c720G-A and c314insT were detected in Ks-2 gene. All of these variants were homozygous in both patients and controls.

CRYM (mu-crystalline homologue-NADP-regulated thyroid hormone binding protein), UniGene Cluster Hs.924, is responsible for most of the intracellular high-affinity $\mathrm{T} 3$ and T4 binding in the kidney and other thyroid hormone-responsive tissues. Thyroid hormone homeostasis plays an important role in kidney growth, renal haemodynamic, tubular function and electrolyte excretion, and 
E2

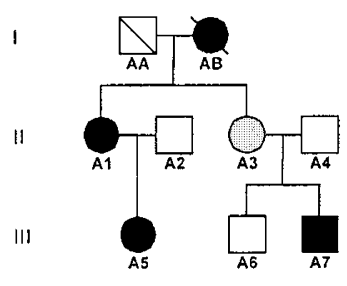

GB8

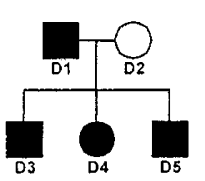

III

IV

E3

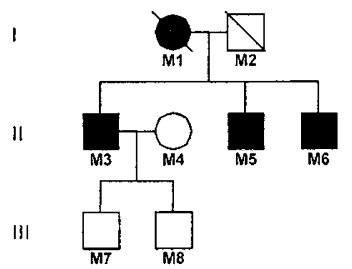

E1

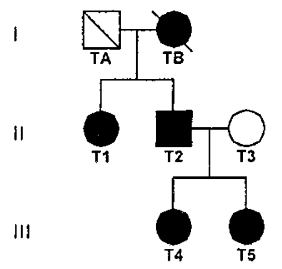

IV

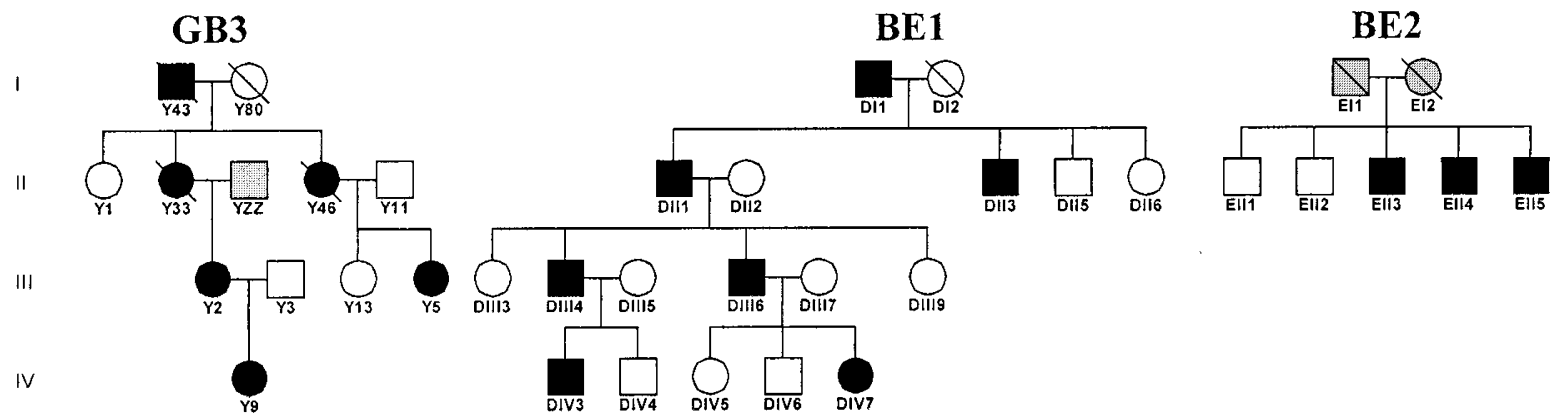

\section{GB2}

GB7

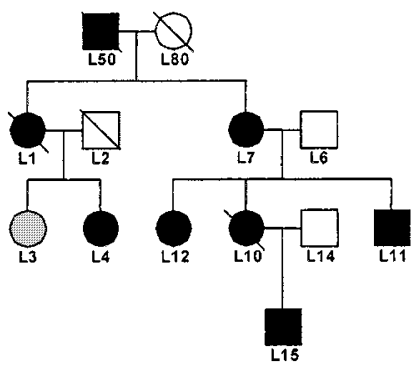

GB6
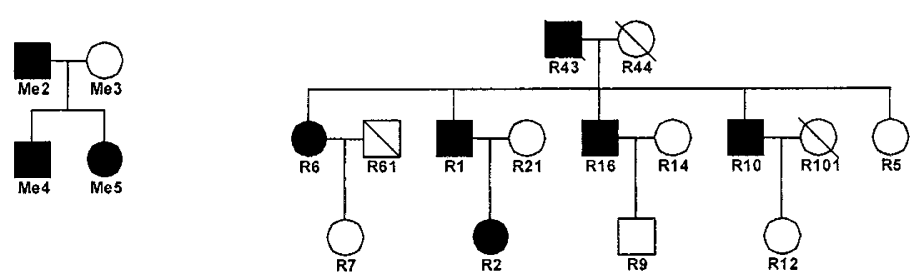

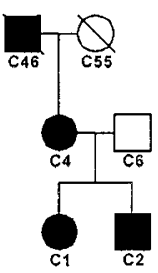

Figure 2 Pedigree diagrams of the investigated families. 


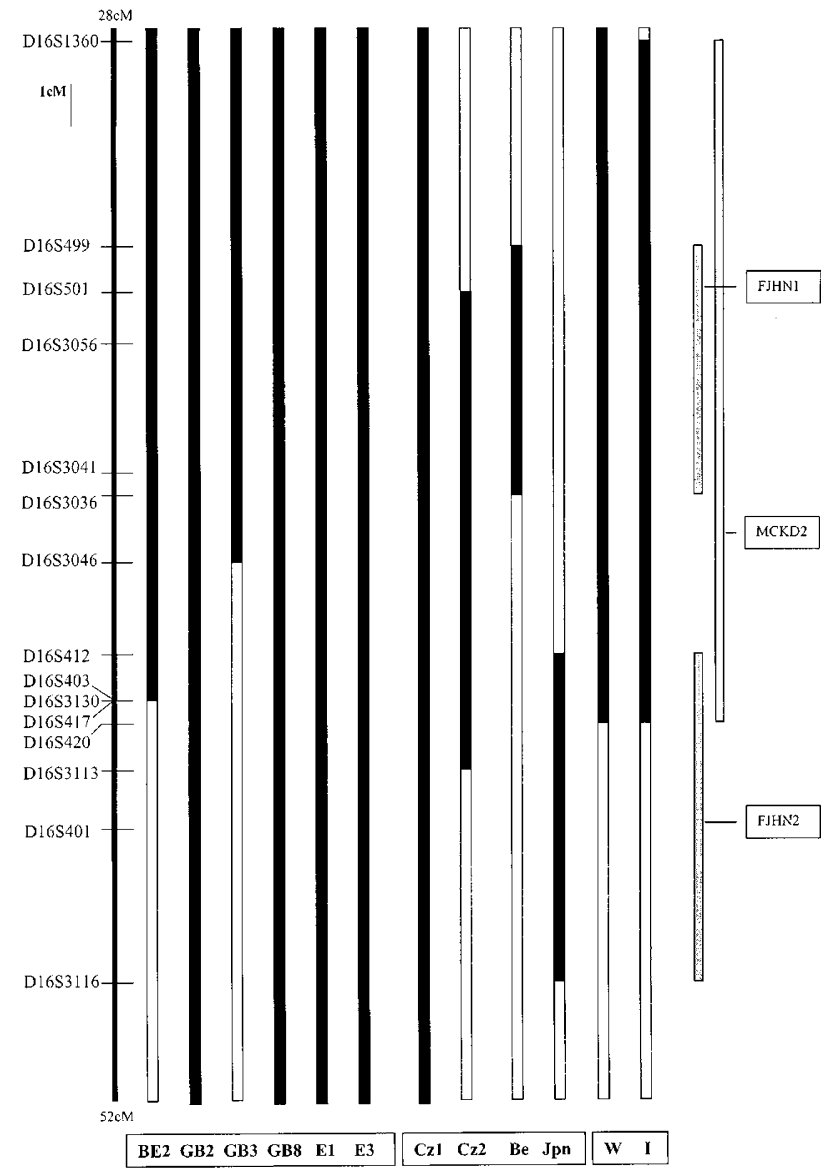

Figure 3 Genetic map of the FJHN critical region on chromosome $16 \mathrm{p} 11.2$ showing schematic representation of crucial recombination events (full bars) detected in six FJHN families from this study (BE2, GB2, GB3, GB8, E1 and E3), in four FJHN families ( $\mathrm{C} z 1$ and $\mathrm{Cz} 2{ }^{32} \mathrm{Be}^{11}$ and $\mathrm{Jpn}^{33}$ and two MCKD2 families $\left(\mathrm{W}^{25}\right.$ and $\mathrm{I}^{34}$ ) from studies reported earlier. Implicated critical region FJHN1, FJHN2 and MCKD2 are shown as grey bars.

association of hyperuricaemia with both hypothyroidism ${ }^{42}$ and hyperthyroidism ${ }^{43}$ has been reported. Three single base pair substitutions located in non-coding regions were detected in the CRYM gene. Two heterozygous substitutions, g2395C-G and g2646T-C, and one homozygous transition g16287T-C were found in both patients and controls.

KIAA1203 UniGene Cluster Hs.16953 is expressed in kidney and contains ubiquitin carboxyl-terminal hydrolase family two domain which may function, through proteosomal pathway, as a regulator of the amiloride-sensitive epithelial $\mathrm{Na}(+)$ channel SCNN1, ${ }^{44}$ whose two subunits, SCNN1B and SCNN1G are located in KIAA1203 in close proximity on $16 \mathrm{p} 11.2$. Three single base pair substitutions were found in the KIAA1203 gene. The transversion c1463T-G changing 488Leu-Arg was found to be both homo-

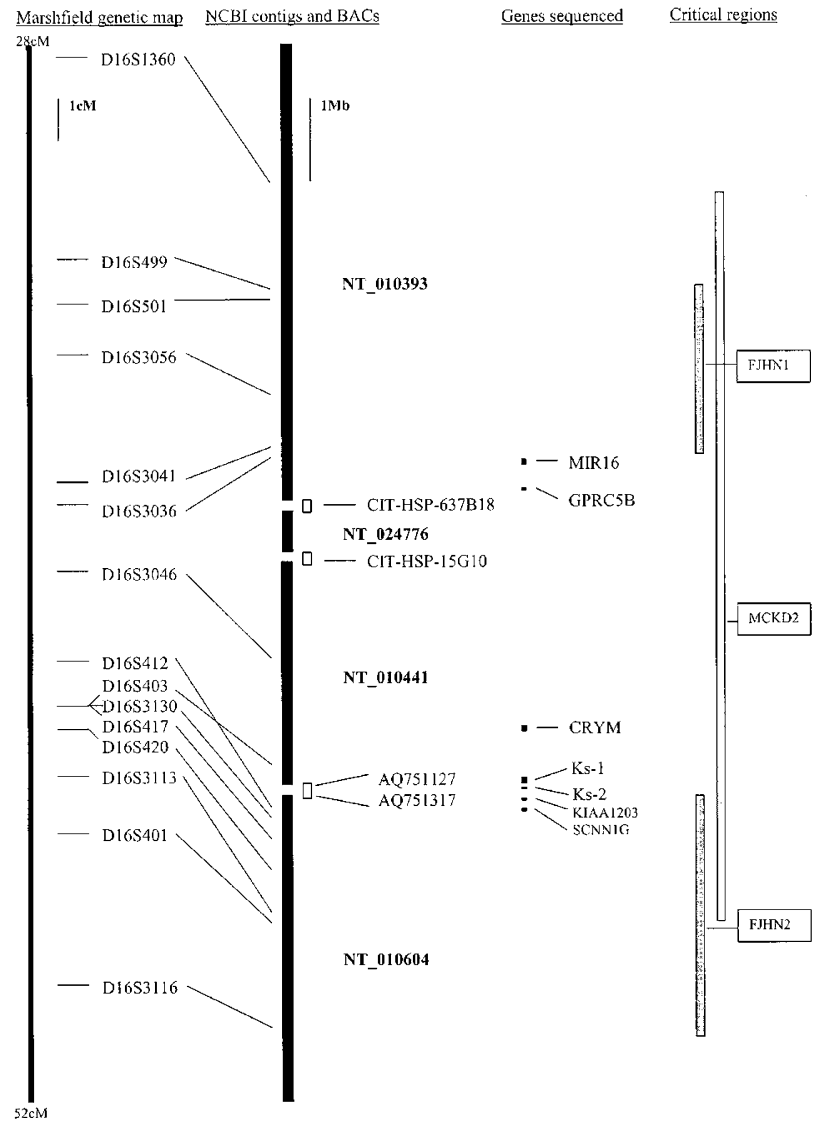

Figure 4 Integrated genetic, physical and transcription map of the FJHN/MCKD2 locus. Genomic contigs are annotated as in NCBI MapViewer. Gapping BAC clones are annotated by their names or BAC end sequence accession.

zygous and heterozygous in patients and controls. The transition c12476G-A changing 822Asp-Asn was homozygous in one of the controls. The transition g14188G-A was found to be heterozygous in both patients and controls.

SCNN1G (gamma subunit of the amiloride-sensitive epithelial sodium channel ENaC), UniGene Cluster Hs.3112, is a protein which mutations result in Liddle's syndrome or pseudohypoaldosteronism type I. It is suggested that variants in this gene may play a role in the pathogenesis of more common forms of human hypertension. ${ }^{45}$ Twenty sequence variants were found in SCNN1G gene. Three resulted in amino acid substitution c532T-A (178Trp-Arg), c1504C-G (502Pro-Ala) and c1840T-C (614Ser-Ala), and were found to be homozygous in both patients and controls. Five heterozygous silent transitions, c387T-C, c474T-C, c549C-T, c636C-T, and a homozygous c825T-C transition were in the coding region of the gene in both patients and controls. Two homozygous substitutions, $-\mathrm{c} 106 \mathrm{C}-\mathrm{G}$ and $-\mathrm{c} 31 \mathrm{G}-\mathrm{A}$ were found in the $5^{\prime} \mathrm{UTR}$ in both patients and controls. Of 10 substitutions located 
in non-coding region of the gene, six -173ex1G-A, -99ex3T-C, +29ex9T-C +33ex11T-G, -7ex11G-A and $-49 \mathrm{ex} 12 \mathrm{~A}-\mathrm{G}$ were heter-ozygous and four substitutions -93 ex2G-C, -49ex2A-G, -4ex2G-C and +8ex4 were homozygous in both patients and controls. A summary of all the genetic variants found is shown in Table 2 .

\section{Discussion}

Linkage analysis

The results of the linkage analysis confirm the already demonstrated linkage of FJHN to chromosome 16p11.2. However, the results also show that there is significant heterogeneity underlying the disease. On the basis of the

Table 2 Summary of all the genetic variants found in selected candidate genes

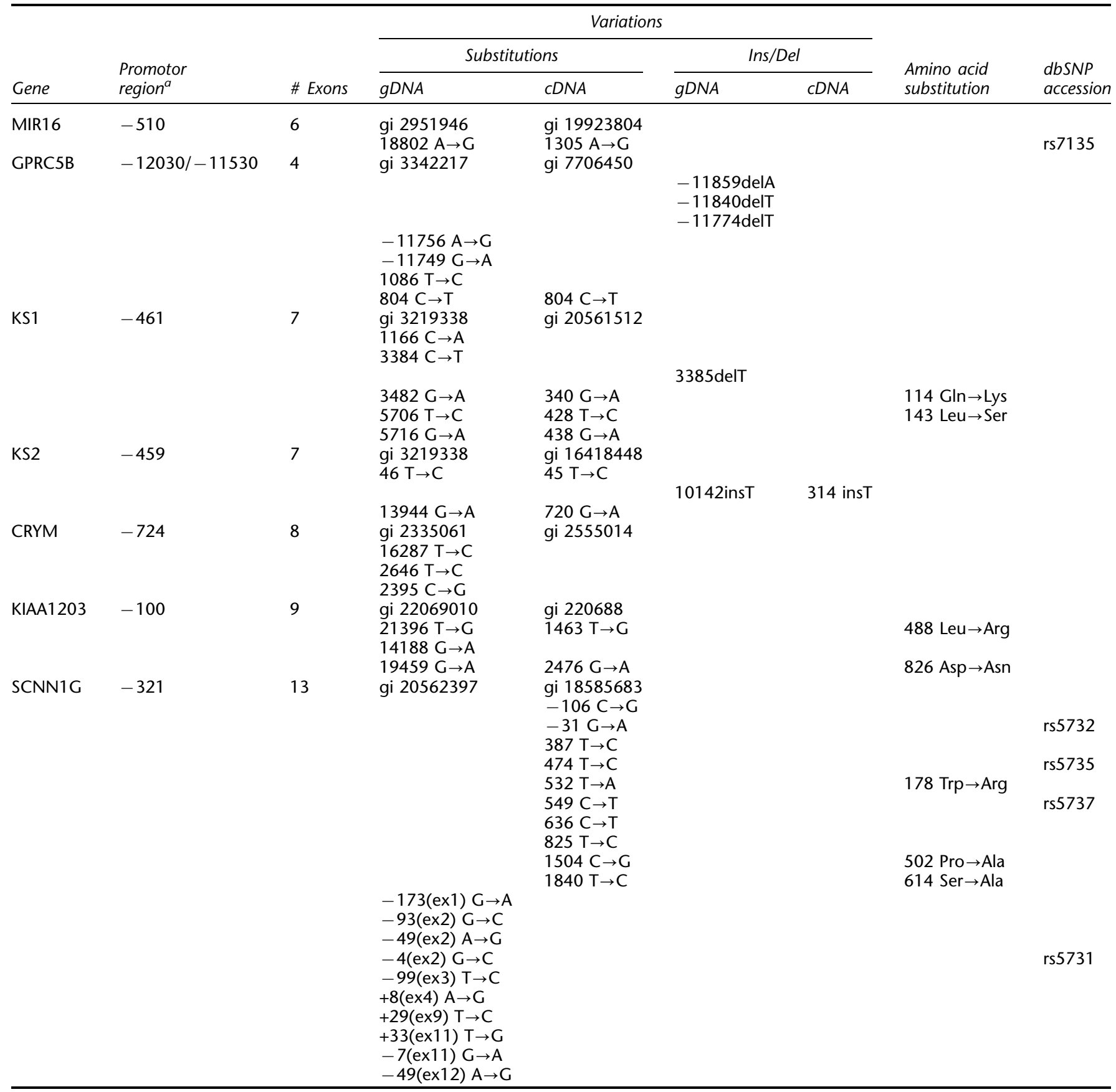

Promotor regions correspond to number of base pairs or genomic region sequenced upstream from the presumed 1st ATG. Annotion of sequence variants is based on their positions respective to the presumed 1st ATG in indicated GenBank sequences. 
maximum HLOD score, we estimate that the proportion $(\alpha)$ of families segregating to a gene linked to $16 \mathrm{p} 11.2$ is 0.37 . However, the parameter $\alpha$ maximised under the hypothesis of heterogeneity should be interpreted as the proportion of individuals in the overall sample that belong in families segregating the linked gene, rather than simply the proportion of families segregating the gene. Hence, the estimate will be influenced by the relative size of families. In our sample, the biggest families (BE1, GB4 and GB6) do not support linkage to $16 \mathrm{p} 11.2$. Effectively, the value of $\alpha=0.37$ is likely to be an underestimate.

\section{Haplotype analysis}

As an alternative approach, investigation of haplotypes segregating within individual families reveals that $40 \%$ of the families have haplotypes consistent with linkage to the investigated region. This value, in turn, is likely to be an overestimate, since some of the families are small and are likely to support linkage by random chance. Nevertheless, by combining the above approaches we are able to estimate that, within our sample, between 37 and $40 \%$ of FJHN cases are explained by linkage to $16 \mathrm{p} 11.2$. In an earlier study ${ }^{32}$ we found that two out of three FJHN families of Czech origin showed linkage of the disease phenotype to 16p11.2. The current results demonstrate that genetic heterogeneity of FJHN is also prevalent in other European populations. Furthermore, our haplotype analysis shows that there is no common haplotype or allele segregating with the disease across families. This suggests that the disease may be the result of several independent de novo mutations, or that the underlying mutation is old and shows no linkage disequilibrium with a particular marker locus. However, since the distance between markers is of the order of $1 \mathrm{cM}$, it is likely that linkage disequilibrium between the causative mutation and the markers may have decayed even for a reasonably young mutation. A much finer marker map would be required to determine which of the above hypotheses is correct.

In addition, we present an up to date synopsis of FJHN linkage results in the context of recently published FJHN/ MCKD2 studies. ${ }^{11,25,32-34}$ The alignment of detected FJHN haplotypes provided two non-overlapping critical regions - FJHN1 delimited by markers D16S499-D16S3036 and FJHN2 delimited by markers D16S412-D16S3116. Out of ten FJHN and two MCKD2 families mapped to $16 \mathrm{p} 11.2$ so far, three FJHN families are linked only to the FJHN1 and one FJHN family only to the FJHN2 regions. The remaining six FJHN and both MCKD2 families reported may be linked to both regions. If FJHN and MCKD2 are two different disease entities then it may be postulated that up to three different genes FJHN1, MCKD2 and FJHN2 may exist in close proximity on chromosome 16. Considering FJHN and MCKD2 as a single disease entity, two loci FJHN1 and FJHN2 may exist. One locus is probable when we omit the results of the Japanese study on the basis of possible inconsistencies in current human genome maps, genotyping errors or existence of phenocopy.

\section{Integrated genetic, physical and transcript map}

Since neither of the hypotheses can be excluded, we focused our attention on the genomic region delimited by markers D16S499 and D16S3116. We have placed all of the Marshfield genetic markers located within the critical region on four genomic contigs. Using BLAST searches we have identified three gap closing BAC clones and obtained thus a contiguous stretch of $7.5 \mathrm{Mbp}$ sequence along which, according to the NCBI annotation, 45 genes and 129 gene-like features are localised.

We have tried to place hypothesis of multiple loci in close proximity associated with similar phenotypes into the context of the critical region gene content. In general, a model of several loci in close proximity can be explained by defects of duplicated genes, genetic defect of proteins comprising multimeric complex (channel, receptor, signalling pathway) and genes responsible for such a complex regulation, processing, trafficking and cell surface stability. Evaluating the genomic structure and gene content, justification for the above hypothesis may be seen in the following features of the critical region; (1) presence of duplicated sequence tracts distributed throughout the parm of chromosome $16 ;{ }^{46}$ (2) co-localisation of the amiloride-sensitive epithelial sodium channel (ENaC) subunits SCNN1B and SCNN1G and of the ENaC possible regulators such as ubiquitin carboxyl-terminal hydrolase KIAA1203, elongation factor-2 kinase and protein kinase PRKCB1; (3) presence of several genes involved in kidney G proteincoupled receptor signalling and microtubule-dependent vesicle transport such as GPRC5B, MIR16, LOC124724, ARL6IP, COG7, GGA, LOC90404, dynein, ERN and RICH1; (4) presence of genes involved in regulated protein degradation such as polo-like kinase PLK, KIAA1203 or LOC123803; and (5) presence of genes involved in podocyte formation - GGA and ARL6IP.

The gene content analysis showed that the delimited chromosomal region contains a number of genes, which may contribute important kidney specific structural units or regulatory functions. Since we have not found evidence for the presence of the urate transporter, we focused on some of the genes which might be involved in G-protein signalling, regulated protein degradation, thyroid hormone homeostasis or which were found to be associated with hypertension.

\section{Candidate gene analyses}

Following the sequence analysis of two genes localised within the critical region - uromodulin and $\mathrm{SAH}^{24}$ we have analysed seven more candidate genes. In all of the genes no 'classic' deleterious mutations were detected within the promotor regions, exon/intron junctions and coding sequences. This finding however cannot completely rule 
out the involvement of those genes in the disease development. The transcription of any of those genes can be greatly affected by mutations in distant promotor regions or by intronic mutations affecting mRNA processing. The cDNA studies, which would be very useful in this respect, could not be performed since we have no affected kidney tissue biopsies available.

In summary, FJHN is a genetically heterogeneous disease. To elucidate its molecular basis, further efforts must be aimed at better clinical characterisation of FJHN/MCKD patients. Affected families must be re-evaluated and followed to determine whether the disease is manageable by allopurinol and whether and when cysts occurred. Importantly, members of all three GB kindreds showing linkage in this report and identified prior to the onset of severe renal disease have been treated successfully with allopurinol - in the case of GB2 and three for from 20 to 37 years. ${ }^{27,28}$ Further gene mapping efforts must be focused on both refining the 16p11.2 locus and discover other FJHN loci in families not linked to 16p11.2. In this regard, it is of interest that kindred GB4, with a history of cysts at biopsy and phenotypically identical with MCKD2, has been found recently (following the onset of diabetes type 2) to have a defect on $17 \mathrm{q}$ involving the hepatocyte nuclear factor $1 \mathrm{~b}$ gene (Bingham et al, personal communication).

Further studies must also be focused on experimentally confined completion of physical and transcript maps of FJHN critical regions and complementary genomic and proteomic approaches. Expression profiling using the microarray technology or 2D gel protein analysis may provide useful information on candidate gene selection and pathogenesis of the disease, and attempts to obtain access to sufficient amount of affected tissues must be undertaken.

The linkage and haplotype analysis reported here further demonstrate the genetic heterogeneity of FJHN and imply the hypothesis of several loci located in close proximity on $16 \mathrm{p} 11.2$ as well. This situation greatly hampers the positional cloning effort and indicates that better clinical characterisation and functional approaches must be undertaken. The report of integrated physical and mostly in-silico predicted transcription maps of the FJHN critical region provides basis for precise experimental annotation of the current transcript map, which is essential for final identification of the FJHN gene(s).

\section{Acknowledgements}

This work was supported by grant 5NE/7046 from the Grant Agency of Ministry of Health and partly, by grants VZ-111100003 from Ministry of Education of the Czech Republic, grant HGOOOOS from the U.S. Human Genome Research Institute and grant 00/350 from the Fondo de Investigaciones Sanitaris.

\section{References}

1 Duncan H, Dixon A: Gout, familial hyperuricaemia and renal disease. Q J Med 1960; 29: 127-136.

2 Cameron JS, Moro F, Simmonds HA: Gout, uric acid and purine metabolism in paediatric nephrology. Pediatr Nephrol 1993; 7: $105-118$.

3 Van Goor W, Kooiker CJ, Mees EJ: An unusual form of renal disease associated with gout and hypertension. J Clin Pathol 1971; 24: 354-359.

4 Massari PU, Hsu CH, Barnes RV, Fox IH, Gikas PW, Weller JM: Familial hyperuricaemia and renal disease. Arch Intern Med 1980; 140: 680-684.

5 Richmond JM, Kincaid-Smith P, Whitworth JA, Becker GJ: Familial urate nephropathy. Clin Nephrol 1981; 16: 163-168.

6 Leumann EP, Wegmann W: Familial nephropathy with hyperuricaemia and gout. Nephron 1983; 34: 51-57.

7 Puig JG, Miranda ME, Mateos FA et al: Hereditary nephropathy associated with hyperuricaemia and gout. Arch Intern Med 1993; 153: $357-365$.

8 Sebesta I, Krijt J, Pavelka K, Maly J, Simmonds HA, McBride MB: Familial juvenile hyperuricaemic nephropathy in adolescents. Adv Exp Med Biol 1994; 370: 73 - 76.

9 Pavelka Jr K, Sebesta I, Blovska J, Maly J, Chadimova M: [Familial juvenile gouty nephropathy]. Cas Lek Cesk 1996; 135: 668-671.

10 Lhotta K, Gruber J, Sgonc R, Fend F, Konig P: Apoptosis of tubular epithelial cells in familial juvenile gouty nephropathy. Nephron 1998; 79: 340-344.

11 Dahan K, Fuchshuber A, Adamis S et al: Familial juvenile hyperuricaemic nephropathy and autosomal dominant medullary cystic kidney disease type 2: two facets of the same disease? J Am Soc Nephrol 2001; 12: 2348-2357.

12 Korom I, Szabo L, Laszlo A, Dallmann L, Ormos J: [Infantile familial hyperuricaemia with gouty manifestations]. Orv Hetil 1979; 120: $589-592$.

13 Murakami T, Kawakami H, Nakatsuka K, Jojima K, Nohno H, Matsuzaki H: Underexcretory-type hyperuricaemia, disproportionate to the reduced glomerular filtration rate, in two boys with mild proteinuria. Nephron 1990; 56: 439-442.

14 Saeki A, Hosoya T, Okabe $\mathrm{H}$ et al: Newly discovered familial juvenile gouty nephropathy in a Japanese family. Nephron 1995; 70: 359-366.

15 Lam PW, Peh WC: Clinics in diagnostic imaging (2). Juvenile gouty arthropathy with associated nephropathy. Singapore Med J 1995; 36: $85-87$

16 Reiter L, Brown MA, Edmonds J: Familial hyperuricaemic nephropathy. Am J Kidney Dis 1995; 25: $235-241$.

17 Auranen M, Ala-Mello S, Turunen JA, Jarvela I: Further evidence for linkage of autosomal-dominant medullary cystic kidney disease on chromosome 1q21. Kidney Int 2001; 60: 1225-1232.

18 Badilla A, Rojas C: [Familial gout and nephropathy in a young woman. Report of one case]. Rev Med Chil 2001; 129: 666-670.

19 McBride MB, Simmonds HA, Moro F: Familial renal disease or familial juvenile hyperuricaemic nephropathy? J Inherit Metab Dis 1997; 20: 351-353.

20 McBride MB, Rigden S, Haycock GB et al: Presymptomatic detection of familial juvenile hyperuricaemic nephropathy in children. Pediatr Nephrol 1998; 12: 357-364.

21 Lipkowitz MS, Leal-Pinto E, Rappoport JZ, Najfeld V, Abramson RG: Functional reconstitution, membrane targeting, genomic structure, and chromosomal localization of a human urate transporter. J Clin Invest 2001; 107: 1103-1115.

22 Krick W, Wolff NA, Burckhardt G: Voltage-driven p-aminohippurate, chloride, and urate transport in porcine renal brush-border membrane vesicles. Pflugers Arch 2000; 441: 125-132.

23 Hildebrandt F, Omram H: New insights: nephronophthisismedullary cystic kidney disease. Pediatr Nephrol 2001; 16: $168-$ 176. 
24 Pirulli D, Puzzer D, De Fusco M et al: Molecular analysis of uromodulin and SAH genes, positional candidates for autosomal dominant medullary cystic kidney disease linked to $16 \mathrm{p} 12$. $J$ Nephrol 2001; 14: 392-396.

25 Hateboer N, Gumbs C, Teare MD et al: Confirmation of a gene locus for medullary cystic kidney disease (MCKD2) on chromosome 16p12. Kidney Int 2001; 60: 1233-1239.

26 Moro F, Simmonds HA, Cameron JS et al: Does allopurinol affect the progression of familial juvenile gouty nephropathy? Adv Exp Med Biol 1991; 309A: 199-202.

27 McBride MB, Simmonds HA, Ogg CS et al: Efficacy of allopurinol in ameliorating the progressive renal disease in familial juvenile hyperuricaemic nephropathy (FJHN). A six-year update. Adv Exp Med Biol 1998; 431: 7-11.

28 Fairbanks L, Cameron J, Venkat-Raman G et al: Early treatment with allopurinol in familial juvenile hyperuricaemic nephropathy (FJHN) ameliorates progression of renal disease in long-term studies. QJM 2002; 95: 597-607.

29 Miranda ME: The influence of allopurinol on renal deterioration in familial nepropathy associated with hyperuricaemia (FNAH). The Spanish Group for the Study of FNAH. Adv Exp Med Biol 1994; 370: 61-64.

30 Lewis EJ, Hunsicker LG, Clarke WR et al: Renoprotective effect of the angiotensin-receptor antagonist irbesartan in patients with nephropathy due to type 2 diabetes. N Engl J Med 2001; 345: $851-860$.

31 Brenner BM, Cooper ME, de Zeeuw D et al: Effects of losartan on renal and cardiovascular outcomes in patients with type 2 diabetes and nephropathy. $N$ Engl J Med 2001; 345: 861-869.

32 Stiburkova B, Majewski J, Sebesta I, Zhang W, Ott J, Kmoch S: Familial juvenile hyperuricaemic nephropathy: localization of the gene on chromosome 16p11.2-and evidence for genetic heterogeneity. Am J Hum Genet 2000; 66: 1989-1994.

33 Kamatani N, Moritani M, Yamanaka H, Takeuchi F, Hosoya T, Itakura M: Localization of a gene for familial juvenile hyperuricaemic nephropathy causing underexcretion-type gout to 16 p12 by genome-wide linkage analysis of a large family. Arthritis Rheum 2000; 43: 925 - 929.

34 Scolari F, Puzzer D, Amoroso A et al: Identification of a new locus for medullary cystic disease, on chromosome 16p12. Am J Hum Genet 1999; 64: 1655-1660.
35 O'Connell JR, Weeks DE: PedCheck: a program for identification of genotype incompatibilities in linkage analysis. Am J Hum Genet 1998; 63: 259-266.

36 Gudbjartsson DF, Jonasson K, Frigge ML, Kong A: Allegro, a new computer program for multipoint linkage analysis. Nat Genet 2000; 25: $12-13$.

37 Kmoch S, Hartmannova H, Stiburkova B, Krijt J, Zikanova M, Sebesta I: Human adenylosuccinate lyase (ADSL), cloning and characterization of full-length cDNA and its isoform, gene structure and molecular basis for ADSL deficiency in six patients. Hum Mol Genet 2000; 9: 1501-1513.

38 Terwilliger J, Ott J: Handbook of human genetic linkage. Baltimore, London: Johns Hopkins University Press, 1994.

39 Zheng B, Chen D, Farquhar MG: MIR16, a putative membrane glycerophosphodiester phosphodiesterase, interacts with RGS16. Proc Natl Acad Sci USA 2000; 97: 3999-4004.

40 Robbins MJ, Michalovich D, Hill J et al: Molecular cloning and characterization of two novel retinoic acid-inducible orphan Gprotein-coupled receptors (GPRC5B and GPRC5C). Genomics 2000; 67: 8-18.

41 Fujino T, Takei YA, Sone $\mathrm{H}$ et al: Molecular identification and characterization of two medium-chain acyl-CoA synthetases, MACS1 and the Sa gene product. I Biol Chem 2001; 276: $35961-35966$

42 Klein I, Levey GS: Unusual manifestations of hypothyroidism. Arch Intern Med 1984; 144: 123-128.

43 Sato A, Shirota T, Shinoda T et al: Hyperuricaemia in patients with hyperthyroidism due to Graves' disease. Metabolism 1995; 44: 207-211.

44 Malik B, Schlanger L, Al-Khalili O et al: Enac degradation in A6 cells by the ubiquitin-proteosome proteolytic pathway. J Biol Chem 2001; 276: $12903-12910$.

45 Iwai N, Baba S, Mannami T et al: Association of sodium channel gamma-subunit promoter variant with blood pressure. Hypertension 2001; 38: 86-89.

46 Loftus BJ, Kim UJ, Sneddon VP et al: Genome duplications and other features in $12 \mathrm{Mb}$ of DNA sequence from human chromosome 16p and 16q. Genomics 1999; 60: 295-308. 